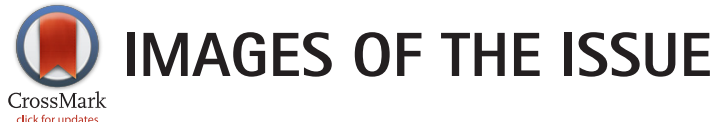

INTESTINAL

ISSN 1598-9100(Print) • ISSN 2288-1956(Online)

RESEARCH

http://dx.doi.org/10.5217/ir.2015.13.3.291

Intest Res 2015;13(3):291-293

\section{A Patient With Abdominal Pain and Fever}

\author{
Yoon Suk Jung \\ Department of Internal Medicine, Kangbuk Samsung Hospital, Sungkyunkwan University, School of Medicine, Seoul, Korea
}

Question: A 72-year old woman presented to our hospital because of severe pain in the right lower quadrant of the abdomen and fever for one day. Her medical history did not involve any serious illness or surgical procedure besides hypertension.
At admission, her blood pressure, pulse rate, respiratory rate, and body temperature were 100/60 $\mathrm{mmHg}$, 64 beats/ min, 20 breaths $/ \mathrm{min}$, and $38.1^{\circ} \mathrm{C}$, respectively. The patient showed signs of acute abdomen, and physical examination revealed direct and rebound tenderness in the right lower
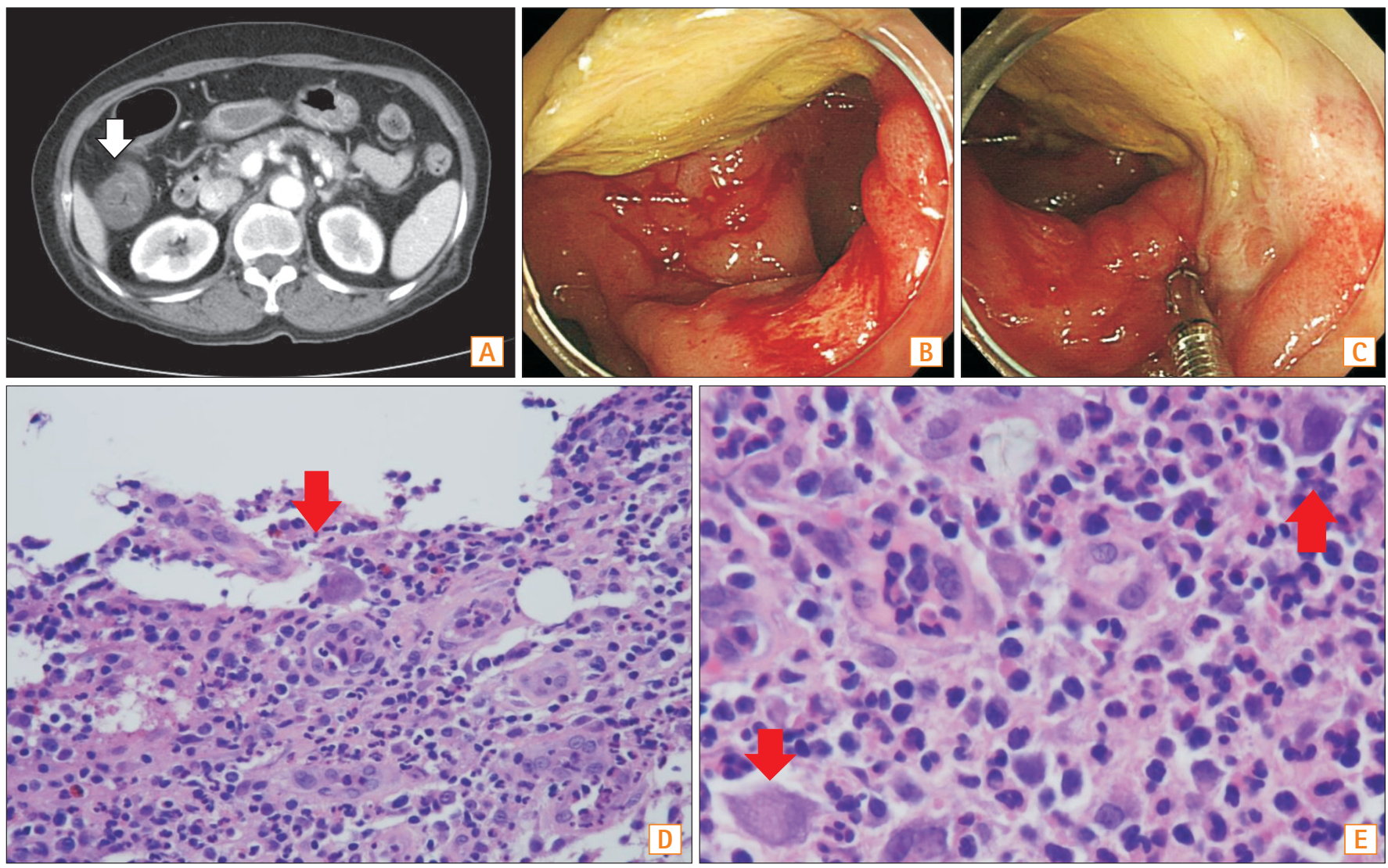

Received April 30, 2015. Revised May 3, 2015. Accepted May 4, 2015.

Correspondence to Yoon Suk Jung, Department of Internal Medicine, Kangbuk Samsung Hospital, Sungkyunkwan University School of Medicine, 29 Saemunan-ro, Jongno-gu, Seoul 110-746, Korea. Tel: +82-2-2001-8577, Fax: +82-2-2001-2049, E-mail: ys810.jung@samsung.com

Financial support: None. Conflict of interest: None. 

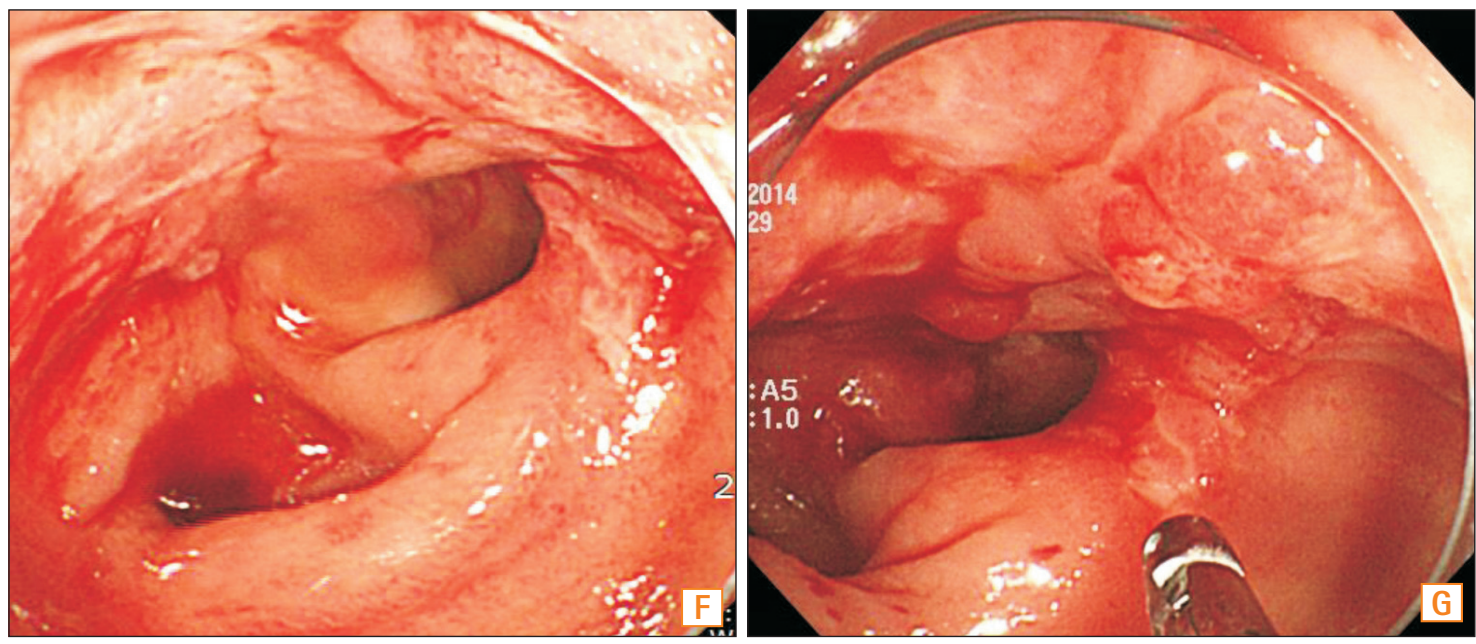

quadrant. Subsequent blood tests showed the following results: white blood cell count and CRP level as $16,600 / \mathrm{mm}^{3}$ (neutrophil segment 91.7\%) and $30.32 \mathrm{mg} / \mathrm{dL}$, respectively. Abdominal CT showed edematous wall thickening and poor bowel wall enhancement in the ascending colon (white arrow in Fig. A), suggesting ischemic colitis or other colitis.

The patient underwent colonoscopy to assess the cause of lesion in the ascending colon. The region from the proximal ascending colon to the cecum showed a large ulceration covered with yellowish exudate. The ulceration was deep, with a clear boundary and accompanied by rubefaction (Fig. $\mathrm{B}, \mathrm{C}$ ). The base and boundary of the ulceration were biopsied, and the pathologic findings are shown in Fig. D and E (red arrow). What is the most likely diagnosis?

\section{Answer to the Images: Cytomegalovirus (CMV) Colitis}

Most cases of CMV colitis occur in immunocompromised patients such as those with congenital or acquired immunodeficiency disease, those receiving immunosuppressive drugs, and those who have undergone transplantations. ${ }^{1}$ However, in rare cases, CMV colitis can also occur in immunocompetent hosts and can cause significant morbidity and mortality. ${ }^{1}$ CMV colitis in immunocompromised patients is usually secondary to the reactivation of latent infection, whereas its occurrence in immunocompetent hosts is usually related to primary infection. ${ }^{2}$ Abdominal pain, diarrhea, and fever are the common presenting symptoms of CMV colitis. $^{3}$ A retrospective study of 12 immunocompetent adults with CMV colitis reported that well-demarcated ulcerations (50\%), ulceroinfiltrative growth (25\%), and pseudomembrane formation (25\%) were the most commonly identified endoscopic abnormalities. ${ }^{4}$ In agreement, the endoscopic findings of this case reported a well-demarcated ulceration. The diagnosis of CMV colitis is based on serology, CMV antigen testing, and colonscopy with biopsy. ${ }^{5}$ The pathologic findings distinctly indicated CMV colitis, as they revealed viral inclusions of the Cowdry owl eye inclusion bodies characteristic of $\mathrm{CMV}^{6}$ The ulcer biopsy of this case showed inflammation of the granulation tissue with occasional CMV-like viral inclusions (Fig. E, F). In addition, simple haematoxylin and eosin staining revealed a few scattered positive cells, representing CMV colitis. Most cases of CMV infection in immunocompetent hosts are associated with minimal or no symptoms, and antiviral therapy is not usually recommended. ${ }^{6}$ However, several case reports have documented successful therapy for immunocompetent hosts with severe manifestations of CMV infection. ${ }^{4,6}$ In our case, the patient was treated with an antiviral therapy (ganciclovir), as she suffered from severe abdominal pain and had a large ulceration, as observed on colonoscopy. After 2 weeks of antiviral therapy, the patient's condition clinical symptoms resolved, and on follow-up colonoscopy, the large ulceration had resolved and the CMV-like viral inclusions were absent on histology (Fig. F, G). The antiviral therapy with ganciclovir appears appropriate for severe CMV colitis even in immunocompetent hosts. This case study showed that despite aggressive clinical manifestation, an immunocompetent host has a good prognosis of CMV colitis, if the infection is diagnosed and treated early.

\section{REFERENCES}

1. Siegal DS, Hamid N, Cunha BA. Cytomegalovirus colitis mimicking ischemic colitis in an immunocompetent host. Heart Lung 2005;34:291-294. 
2. Just-Nübling G, Korn S, Ludwig B, Stephan C, Doerr HW, Preiser W. Primary cytomegalovirus infection in an outpatient setting-laboratory markers and clinical aspects. Infection 2003;31:318-323.

3. Klauber E, Briski LE, Khatib R. Cytomegalovirus colitis in the immunocompetent host: an overview. Scand J Infect Dis 1998;30:559-564.

4. Seo TH, Kim JH, Ko SY, et al. Cytomegalovirus colitis in immunocompetent patients: a clinical and endoscopic study. Hepatogastroenterology 2012;59:2137-2141.
5. Goodman AL, Murray CD, Watkins J, Griffiths PD, Webster DP. CMV in the gut: a critical review of CMV detection in the immunocompetent host with colitis. Eur J Clin Microbiol Infect Dis 2015;34:13-18.

6. Lancini D, Faddy HM, Flower R, Hogan C. Cytomegalovirus disease in immunocompetent adults. Med J Aust 2014;201:578580. 